\title{
Parametric Extraction of the Road Conditions Spatial Data and Detection of Defeats using Pragmatic Clustering Method
}

\author{
Dara Anitha Kumari, A. Govardhan
}

\begin{abstract}
Growth in the population and road transportation for any region can create a higher demand for better road conditions and the less safe road conditions can be a great bottleneck for the growth of the nation. Hence most of the progressive nations like India,builds better infrastructure for the road transportations.Nevertheless, the higher populations in countries like India,demand greater maintenance of the roads and for a gigantic demographic, the maintenance work can be very tedious. Also,the damage in the road surfaces can also lead to the increasing rate of the accidents. Henceforth,the demand of the road maintenance is always increasing. Nevertheless, the existing road maintenanceauthorities deploy a manual process for identification of the repair needs, which is naturally highly time consuming. Thus, this work identifies the demand for automation of the road condition monitoring system and identifies the defects based on three classes as cracks, patch works and potholes on the road surface. The work deploys a novel model for parametric extraction, in order to segregate the defect types. The segregation of the defect types can be highly challenging due to the nature of the data, which clearly hints to solve the problem using unsupervised methods. Thus, this work also deploys a pragmatic clustering method using a decisive factor, which is again generated from the extracted features of parameters. The work demonstrates nearly $96 \%$ accuracy on the benchmarked dataset with sophistication on the complexity of the model.
\end{abstract}

Keywords: Road condition identification, Pragmatic Clustering, Parametric Analysis,intensity Variation, Coordinate Mapping, Segmentation.

\section{INTRODUCTION}

The road conditions in any country or region are one of the crucial factors for the transportation of people or goods and directly influence the economy. The report presented by European commission et al. [1] on 2018 have significantly demonstrated that small deviation of the road condition can lead to fatal accidents.Moreover, the defected road surfaces can also cause problem to the vehicles, especially to the components like break, suspension and drive wheels.Hence the growing countries like India puts some great efforts to maintain the road surfaces cleaned from defects.

Revised Manuscript Received on February 05, 2020.

* Correspondence Author

Anitha Kumar Dara, Department of Computer Science and Engineering, JNTUH CEH, Hyderabad, Telangana, India .

Dr.A.Govardhan, Professor of Computer Science \& Engineering, Registrar and Executive Council Member, Jawaharlal Nehru Technological University Hyderabad (JNTUH). India.

(C) The Authors. Published by Blue Eyes Intelligence Engineering and Sciences Publication (BEIESP). This is an open access article under the CC BY-NC-ND license (http://creativecommons.org/licenses/by-nc-nd/4.0/)
Yet another report from the C. N. do Transports[2] also reported that the deformation in the road conditions can lead to increase in the safety hazards from $25 \%$ to $50 \%$ in one single year 2010. Thus, the regular monitoring of the road conditions must increase.

Henceforth, realizing the increasing demand and increasing safety concerns, many of the researches have attempted to detect the road surface defects by various means. The manual process of detecting the road conditions is highly difficult and relies on the human efforts. Henceforth, the researchers are aiming to automate the detection process and the work by A. Fox[3] is the first of its kind to automate the process. This work deploys the detection of the road defects using vehicle mounted sensors and the further process the data in order to detect the defects. The outcome from this work have significantly changed the way this research problem was viewed in the past and number of researchers have shifted their attention towards the information extraction-based solution to this problem. Nevertheless, the identification of the defects is not sufficient in order to proceed for the proper repair works. Thus, classification of the road condition defects such as cracks, patchworks or potholes must be detected.

Henceforth, this work demonstrates a novel outcomebased model for extraction of the parameter and further builds pragmatic clustering solution to identify the type and severity of the defects as well.

The rest of the work is elaborated as in section - II, the traditional clustering method is elaborated and the subsequent problems are identified, in section - III, parallel research outcomes are analysed with the gaps in the results, in section -IV, the problem is identified and formulated, in section $-\mathrm{V}$, the proposed solution is formulated using mathematical modelling, in section -VI, the proposed algorithm is discussed, the results are furnished in section VII, the comparative analyses is carried out in section VIII, and the work presents the final conclusion in the section - IX.

\section{TRADITIONAL CLUSTERING METHOD}

In this section of the work, the traditional clustering method as fundamental is discussed. The fundamental understanding of the existing traditional system is important as this helps in the realizing the advancements made to the fundamental principle by the parallel research works. 
Firstly, assuming that, $\mathrm{D}[]$ is the initial dataset and it is a collection of multiple attributes forming the data domain, where each and every attribute can be denoted as $\mathrm{A}_{\mathrm{x}}$. Thus, for a total of $n$ number of attributes, this can be formulated as

$$
D[] \Rightarrow \sum_{i=1}^{n} A_{i}
$$

Further, each attribute $A_{x}$ mustbe analysed for the data domain,

$R\left(A_{x}\right)$, and for a total of $r$ number of total observations, this relation can be noted as,

$$
A_{X} \Rightarrow \sum_{j=1}^{r} \mathfrak{R}\left(A_{X}\right)[j]
$$

Further, one of the attributes must be identified as the class attribute, Ac , in the dataset and the other attributes must be mapped against the class attributes in order to form the clusters.

Assuming that, the distribution of the data for any attributes then the data domain for the attributes can be separated in three segments as,

$$
C_{p}=\int_{j=1}^{r}\left|\mathfrak{R}\left(A_{X}\right)[j] \rightarrow \lambda\right|
$$

Here, $\lambda$ denotes the central tendency for the quartile.

Henceforth, for each attributes the formation of the clusters must be realized, and any sample cluster can be visualized as following [Fig-1].

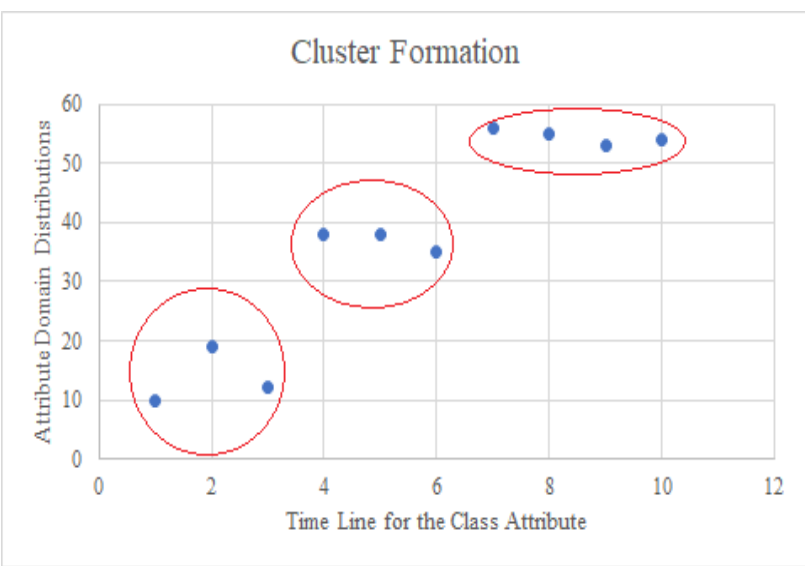

Fig 1: Sample Cluster Formulations

This fundamental knowledge will help in realizing the advancements studied in the next section of the work for the parallel research outcomes.

\section{PARALLEL RESEARCH OUTCOMES}

After the realization of the fundamental principle of the process, in the section of the work, the parallel research outcomes are analysed in detail.

The work outcome by T. Kim [4] have significantly demonstrated and comparative studies from various other research attempts. From this work, the conclusive statement can be generated as, the gaps in the research is existing and further elaborations of the solution is a must. Also, the parallel research works majority focuses on the twodimensional dataset, whereas the demand from the trends $A_{x}$, can be observed to have three similarity distribution ,

are towards the spatial data. The similar problem solution was proposed using machine learning approaches by S.Karabachos . [5]which is again a land mark for this domain of the research as the reduction is very high.

In the recent times, the popularity of the automated vehicles are also increasing and the demand for avoidance of the defected surface is gaining. thus, the extraction of the parametric observation for the road surface defects is one of the prime tasks for this domain of research. The work by $\mathrm{H}$. Bello - Salau [6] have showcased the solution specific extraction of the parameter are possible and further the solution accuracy can be improved to a greater extend. The applicability of the wavelet-based solutions can directly be applied for the purposed [7] as demonstrated by F. M. Bayer [8].

In the other hand, many of the research attempts such as the work by K. Azhar [9] focuses on the traditional image processingmechanisms and further extraction of the parameter some of the other research attempts like the work by P. Wang [10] have applied segmentation methods for identification of the defect objects on the image.

Few of the research attempts have demonstrated the application of neural neural networks such as the work by $\mathrm{H}$. Maeda [11] and achieved a higher accuracy. However, these works had to compromise on the time complexity for the initial data processing tasks.

Henceforth, with the detailed understanding of the parallel research outcomes, this work proposes for formulation of the problem in the next section of the work.

\section{IDENTIFICATION OF THE PROBLEM}

After the detailed understanding of the parallel research outcomes, in this section of the work, the research problem is identified. The fundamental problems are identified based on the parallel research outcomes are two as for detection of the defects on the road surface are critical and sufficient parameters must be identified and, in the situations, where the unavailability of the class attributes ca lead to unsupervised learning methods, the collaborative formulation must be carried out to collaborate all the attributes.

\section{A. Insufficient Extraction of the parameter}

Firstly, assuming that the spatial data is denoted by SP[] and each and every component of the dataset must be defined as $D_{x}$. Thus, for a total of $n$ number of attributes, this relation can be formulated as,

$$
S P[]=\sum_{i=1}^{n} D_{i}
$$

Further, the data components must be equipped with the parameters such as segmentation information, SI, distance between the segmentation , DS, Depth, D, Width ,W, and finally the area A.Assuming that, every image , I, can be represented using the collection of pixel vector, $\mathrm{p}[\mathrm{]}] \mathrm{]}$, then for a $n, m$ number of pixels, the relation can be formulated as

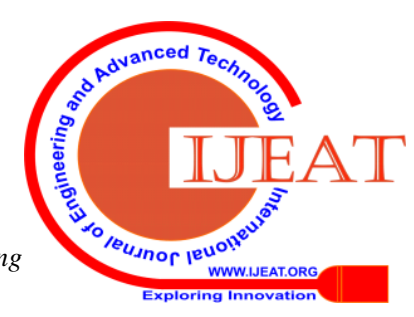


$I=\sum_{i, j}^{n, m} P[i][j]($ Eq.5)

However, in order to extract the SI and A, the boundary information of the image must be extracted using the coordinate information, corr[][], as,

$$
\operatorname{Corr}[][] \Leftarrow P[][]
$$

And

$$
A \Leftarrow \frac{\prod_{\text {Count }} \operatorname{Corr}[][]}{\left|\prod_{\text {Count }} \operatorname{Corr}[][]-P[][]\right|}
$$

Hence, the coordinate information is a must to have for the image information extraction.

Furthermore, in order to calculate the DS and W, the maxima and minima information, max \& min, from the coordinate information is a must to contain, as,

$\max \Leftarrow \frac{\prod_{\text {Length_max }} \operatorname{Corr}[][]}{\operatorname{Corr}[][]}$

And

$\min \Leftarrow \frac{\prod_{\text {Length_min }} \operatorname{Corr}[][]}{\operatorname{Corr}[][]}($ Eq.9)

Thus, the maxima and minima information are must to have for the image information extraction.

Finally, in order to extract the D, the intensity variation, $\mathrm{IV}$, is a must to obtain as,

$D \Leftarrow \frac{I V[]}{\Delta t}$

Based on the parallel research outcomes, it is been observed that these expected parameters are not been considered during the parametric extraction of the road condition.

Thus, this work elaborates on the extraction of the demonstrated and identified parameters.

\section{B. Unavailability of the determiningfactors}

Secondly, during the clustering process, the validity of the clusters is highly difficult as the parameters are different in nature and mostly the parameters multivariate.

Multivariate examination can be entangled by the craving to incorporate material science-based investigation to compute the impacts of factors for a various levelled "arrangement of frameworks". Regularly, examines that desire to utilize multivariate examination are slowed down by the dimensionality of the issue. These worries are regularly facilitated using surrogate models, profoundly precise approximations of the material science-based code.

Henceforth, based on the parallel research outcomes, it is been observed that the determining factor based on deep calculation or pragmatic calculation is highly recommended.

Thus, this work demonstrates the pragmatic cluster formation in the next section.

\section{PROPOSED SOLUTION- MATHEMATICAL MODEL}

After the detailed analysis of the parallel research outcomes and preceded by identification of the problem, in this section of the work the mathematical model is presented.

The work demonstrates the mathematical model for five different components of the solutions here.

LEMMA -1:Extraction of the coordinate values from the pixel information can determine the maximal and minimal distance between the objects in the road condition information.

PROOF:Assuming that spatial data set is denoted as SPD and each and every SPD is collection of the image, $\mathrm{I}_{\mathrm{x}}$ and other data information $\mathrm{D}[]$. Thus, this can be formulated as,

$S P D=\langle I, D[]\rangle$

Further, the image component Can be represented as collection of pixel information,P[][], for $\mathrm{n}$ rows and $\mathrm{m}$ columns. This can be formulated as,

$$
I_{X}=\sum_{i, j}^{n, m} P[i][j]
$$

Assuming that, the image pixels are not converted to the gray scale variation, the image must be converted to the gray variation as

$$
P_{G}[][]=\prod_{(r, g, v) \rightarrow g r a y} P[][]
$$

Henceforth, the image pixels sets are ready to converted into the coordinate mapping into coordinate vector, Corr[][] as,

$$
\operatorname{Corr}[][]=\text { Iff } P_{G}[i][j] \neq \phi
$$

Where, $\phi$ denotes the background pixels or the pixel from the background regions.

Henceforth, for the image objects, $I_{1}$ and $I_{2}$ are converted into two different coordinate vectors as $\operatorname{Corr}_{1}[]$ and $\operatorname{Corr}_{2}[]$, as

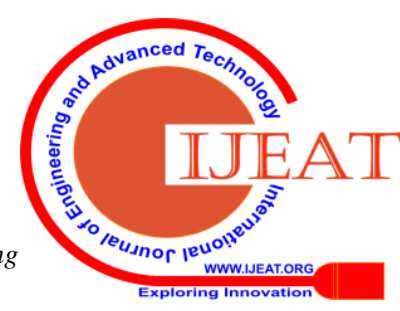




$$
\operatorname{Corr}_{1}[][] \leftarrow I_{1}
$$

And,

$$
\operatorname{Corr}_{2}[][] \leftarrow I_{2}
$$

Further, the locations maxima, max1 and max2 and the location minima, $\min _{1}$ and $\min _{2}$ can be formulated as

$$
\max _{1}=\prod_{\max } \operatorname{Corr}_{1}[][](\text { Eq.17) }
$$

And

$$
\max _{2}=\prod_{\max } \operatorname{Corr}_{2}[][]
$$

With,

$$
\min _{1}=\prod_{\min } \operatorname{Corr}_{1}[][]
$$

And,

$$
\min _{2}=\prod_{\min } \operatorname{Corr}_{2}[][]
$$

Further, the actual minima, $\alpha$, and actual maxima, $\beta$ from the coordinate vector can be extracted using the mapping method [Fig-2] as,

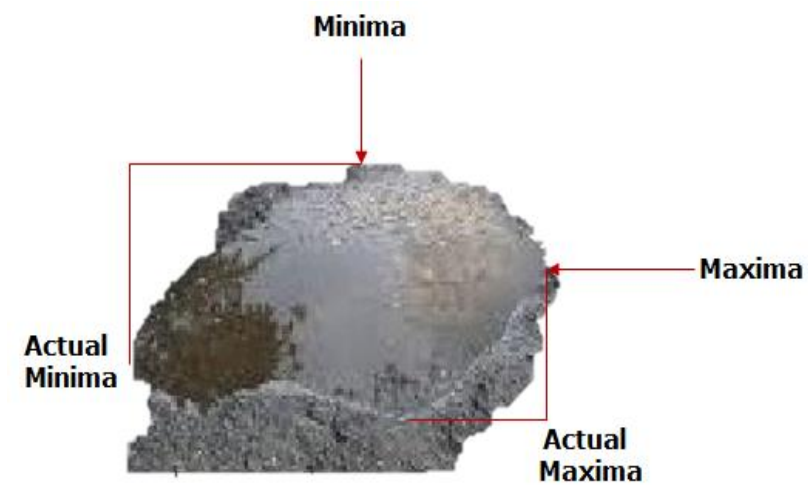

Fig. 1 Minima and Maxima Mapping from Coordinate Vector

$$
\alpha_{1}=\prod_{\min _{1}(0, m) \cap \max _{1}(0, m)} \operatorname{Corr}_{1}[][] \quad \text { (Eq.21) }
$$

And,

$$
\alpha_{2}=\prod_{\min _{2}(0, m) \cap \max _{2}(0, m)} \operatorname{Corr}_{2}[][] \text { (Eq.22) }
$$

With

$$
\beta_{1}=\prod_{\min _{1}(n, 0) \cap \max _{1}(n, 0)} \operatorname{Corr}_{1}[][]
$$

And

$$
\beta_{2}=\prod_{\min _{2}(n, 0) \cap \max _{2}(n, 0)} \operatorname{Corr}_{2}[][]
$$

Thus, the distance vector for minimal distance, DS $_{\text {Min }}$ and distance vector for maximum distance $\mathrm{DS}_{\operatorname{Max}}$ can be formulated as
$D S_{\text {Min }}=\left|\alpha_{1}-\alpha_{2}\right|$

And

$$
D S_{\text {Max }}=\left|\beta_{1}-\beta_{2}\right|
$$

Thus, the extraction of the minimum and maximum distance is completed the results obtained from this algorithm is elaborated in the further section of this work.

LEMMA-2:Extraction of the intensity variation can produce the depth of the defects using the centroid estimation in the road condition information.

PROOF:Assuming that spatial data set is denoted as SPD and each and every SPD is collection of the image, Ix and other data information D[]. Thus, this can be formulated as,

$S P D=\langle I, D[]\rangle$

(Eq.19) Further, the image component can be represented as collection of pixel information, $\mathrm{P}[][]$, for $\mathrm{n}$ rows and $\mathrm{m}$ columns. This can be formulated as,

(Eq.20) $I_{X}=\sum_{i, j}^{n, m} P[i][j]$

Assuming that,the image pixels are not converted to the gray scale variations, the image must be converted to the gray variations, as,

$P_{G}[][]=\prod_{(r, g, v) \rightarrow \text { gray }} P[][]$ (Eq.29)

Further, for the centroid detection, the pixel pairs vectors, Pix, Pair[][] is to identified ,as

$$
\underset{\text { (Eq.30) }}{\text { Pix_Pair }[][]} \leftarrow\left|P_{G}[x][y]-P_{G}[x+i][y+j]\right| \neq \phi
$$

Where, $\phi$ denotes the background pixels or the pixel from the background regions

Henceforth, the image pixel sets are ready to be converted into the coordinate mapping into coordinate vector, Corr[][] as,

$$
\text { Corr[][] = Iff Pix_Pair[][] } \neq \phi
$$

Furthermore, here from the Corr[][] vector , the maximum intersection must be identified in order to determine the centroid, $\sigma$ of the image, as by applying convolution techniques

$$
\sigma[][]=\operatorname{ConV}(\operatorname{Corr}[][])
$$

After the centroid extraction, the intensity calculation must be carried for the centroid, maxima region and minima region,as

$$
\begin{aligned}
& \omega_{C}=\iint_{i, j} \sigma[i][j] \\
& \omega_{\text {Min }}=\iint_{i, j} \alpha_{1}[i][j]
\end{aligned}
$$


And

$\omega_{\text {Max }}=\iint_{i, j} \beta_{1}[i][j]$

Where, $\omega_{C}, \omega_{\text {Min }}$ and $\omega_{\text {Max }}$ are the intensity for each of the regions mentioned above.

Further, the intensity variation coefficient, $\eta$ must be calculated as,

$\eta=\frac{1}{\omega_{C}} \cdot \frac{\left|\omega_{M i n}-\omega_{M a x}\right|}{\Delta\left|\omega_{\text {Min }}-\omega_{\text {Max }}\right|}$

Finally, the depth of the defect, $\delta$ can be calculated as,

$$
\delta=\left|\omega_{\text {Min }}-\omega_{C}\right| \cdot \eta
$$

Thus, the extraction of the defect depth is completed. The results obtained from this algorithm is elaborated in the further section of this work.

LEMMA -3: The width of the defect can be calculated using the maxima and minima points in the road condition information

PROOF: Assuming that spatial data set is denoted as SPD and each and every SPD is collection of the image, Ix and other data information D[]. Thus, this can be formulated as,

Further, the image component can be represented as collection of pixel information, $\mathrm{P}[][]$, for $\mathrm{n}$ rows and $\mathrm{m}$ columns. This can be formulated as,

$$
I_{X}=\sum_{i, j}^{n, m} P[i][j]
$$

Assuming that,the image pixels are not converted to the gray scale variations, the image must be converted to the gray variations, as

$$
P_{G}[][]=\prod_{(r, g, v) \rightarrow \text { gray }} P[][]
$$

Henceforth, the image pixel sets are ready to be converted into the coordinate mapping into coordinate vector, Corr[][] as,

$$
\operatorname{Corr}[][]=\text { Iff } P_{G}[i][j] \neq \phi
$$

Where, $\phi$ denotes the background pixels or the pixel from the background regions.

Henceforth, the width, $\varepsilon$, of the defect can be calculated as.

$$
\varepsilon=\left|\prod_{\operatorname{Corr}[\alpha][0]} P_{G}[i][j]-\prod_{C o r r[0][\beta]} P_{G}[i][j]\right| \text { (Eq.42) }
$$

Thus, the extraction of the defect width is completed. The results obtained from this algorithm is elaborated in the further sections of the work.

LEMMA-4: The area of the defect can be calculated using the coordinate vector from the road condition information.

$$
S P D=\langle I, D[]\rangle
$$

PROOF - Assuming that spatial data set is denoted as SPD and each and every SPD is collection of the image, Ix and other data information D[]. Thus,this can be formulated as,

$$
S P D=\langle I, D[]\rangle
$$

Further, the image component can be represented as collection of pixel information, $\mathrm{P}[][]$, for $\mathrm{n}$ rows and $\mathrm{m}$ columns. This can be formulated as,

$I_{X}=\sum_{i, j}^{n, m} P[i][j]$

Assuming that,the image pixels are not converted to the gray scale variations, the image must be converted to the gray variations, as

$$
P_{G}[][]=\prod_{(r, g, v) \rightarrow \text { gray }} P[][](\text { Eq.45) }
$$

Henceforth, the image pixel sets are ready to be converted into the coordinate mapping into coordinate vector, Corr[][] as,

$$
\operatorname{Corr}[][]=\text { Iff } P_{G}[i][j] \neq \phi
$$

Where, $\phi$ denotes the background pixels or the pixel from the background regions.

Finally, the area, A of the defect region can be calculated as

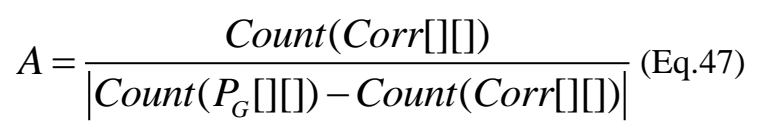

Thus, the extraction of the defect area is completed. The results obtain from this algorithm is elaborated in the further sections of the work.

\section{LEMMA-5:}

In the absence of the class determination factors, the pragmatic calculation of the ratio-based coefficients can form the determining factor.

PROOF :Assuming that, the parametric extraction of the dataset DS[] is resulting into the minimum and maximum distance, $D S_{\text {Min }}$ and $D S_{\text {Max }}$, depth of the defect, $\delta$ width of the defect, $\varepsilon$,and finally area of the defect,A. This can be formulated as

$$
D S[]=\left\langle D S_{\text {Min }}, D S_{M a x}, \delta, \varepsilon, A\right\rangle
$$

Further scaling up the first two parameters as, 
$\frac{\sum_{i=1}^{n} D S_{\text {Min }}}{\sum_{i=1}^{n} D S_{\text {Max }}}=\frac{\beta_{2}}{\beta_{1}}$

(Eq.49)

Or,

$\beta_{1} \cdot D S_{\text {Min }}=\beta_{2} \cdot D S_{\text {Max }}$

(Eq.50)

Again, with the first three parameters,

$\sum_{i=1}^{n} D S_{\text {Min }}: \sum_{i=1}^{n} D S_{\text {Max }}: \delta=\beta_{3}: \beta_{2}: \beta_{1}$

(Eq.51)

Similarity,

$\sum_{i=1}^{n} D S_{\text {Min }}: \sum_{i=1}^{n} D S_{\text {Max }}: \delta: \varepsilon: A=\beta_{5}: \beta_{4}: \beta_{3}: \beta_{2}: \beta_{1}$

(Eq.52)

Thus, the final determining factor, $\Upsilon$ can be formulated as

$\Upsilon=\beta_{0}+\beta_{1} \cdot D S_{\text {Min }}+\beta_{2} \cdot D S_{\text {Max }}$

$+\beta_{3} \cdot \delta+\beta_{4} \cdot \varepsilon+\beta_{5} \cdot A$

(Eq.53)

Henceforth, with the detailed discussion on the mathematical formulation of the proposed solution, in the next section of the work, the algorithm is furnished and discussed.

\section{PROPOSEDALGORITHM -PRAGMATIC CLUSTERING}

Henceforth, with the detailed understanding of the parallel research outcomes, formulation of the problems, and modelling of the proposed solutions, in the section of the work, the proposed algorithm is formulated.

\begin{tabular}{|l}
\hline Algorithm: Parametric Extraction and Pragmatic \\
Clustering for Defect Detection (PE-PC-DD) \\
\hline Step - 1. Accept the road condition information \\
Step - 2. Separate the image and coordinate \\
information as I[] and SP[] \\
Step - 3. For all elements in I[] as I[i] \\
a. Apply gray scaling for I[i] \\
b. Apply segmentation for I[i] and store the \\
objects as Obj[] \\
c. For each element in Obj[] as Obj[j] \\
i. Perform the coordinate mapping of the \\
objects \\
ii. Extract Min and Max coordinate points \\
iii. Calculate the max distance between the \\
objects and store in DS[] \\
iv. Identify the maximum intersection and \\
centroid of the objects \\
v. Calculate the intensity of max, min and
\end{tabular}

Parametric Extraction Results

\begin{tabular}{|c|c|c|c|c|c|c|c|}
\hline $\begin{array}{c}\text { Dataset } \\
\text { Element } \\
\text { ID }\end{array}$ & $\begin{array}{c}\text { Long } \\
\text { Slope }\end{array}$ & $\begin{array}{c}\text { Perpen } \\
\text { dicular } \\
\text { Slope }\end{array}$ & $\begin{array}{c}\text { Defect } \\
\text { Numb } \\
\text { er }\end{array}$ & $\begin{array}{c}\text { Max } \\
\text { Distance of } \\
\text { the Defect } \\
\text { (cm) }\end{array}$ & $\begin{array}{c}\text { Depth } \\
\text { of the } \\
\text { defect } \\
\text { (cm) }\end{array}$ & $\begin{array}{c}\text { Averag } \\
\text { e } \\
\text { Width } \\
\text { (cm) }\end{array}$ & $\begin{array}{c}\text { Area } \\
\left(\mathbf{c m}^{2}\right)\end{array}$ \\
\hline 1 & -0.21 & 4.71 & 1 & 250.46 & 122.23 & 88.89 & 22263 \\
\hline 2 & 0.33 & -3 & 1 & 199.22 & 65.21 & 43.06 & 8579 \\
\hline 3 & -0.21 & 4.7 & 1 & 976.34 & 266.06 & 241.2 & 235494 \\
\hline 4 & -0.3 & 3.29 & 1 & 402.39 & 189.53 & 49.69 & 19994 \\
\hline 4 & -0.15 & 6.78 & 2 & 246.64 & 182.18 & 36.94 & 9110 \\
\hline 4 & 0.23 & -4.3 & 3 & 278.23 & 185.52 & 27.47 & 7644 \\
\hline 5 & 0.65 & -1.54 & 1 & 166.98 & 99.18 & 55.89 & 9333 \\
\hline 6 & 0.72 & -1.4 & 1 & 907.43 & 648.38 & 397.55 & 360747 \\
\hline 7 & 0.6 & -1.67 & 1 & 602.82 & 692.67 & 167.89 & 101208 \\
\hline 7 & 0.78 & -1.28 & 2 & 870.4 & 755 & 308.03 & 268105 \\
\hline 8 & 0.75 & -1.33 & 1 & 1029.8 & 774.36 & 371.21 & 382272 \\
\hline 9 & 0.17 & -5.97 & 1 & 175.41 & 21.17 & 19.46 & 3413 \\
\hline 10 & 0.45 & -2.23 & 1 & 354.05 & 158.68 & 88.54 & 31347 \\
\hline
\end{tabular}

Identify the depth, based on intensity variation and store in DS[]

vii. Calculate the width of the defects from the coordinate mapping information and store in DS[]

viii. Calculate the area using the original and gray scale information and store in DS[]

d. For all elements DS[]

i. Identify the scaling factors

ii. Based on the scaling factors, calculate the determining factor

e. For all elements in DS[]

i. Apply clustering method of determining factor

Table-1 parametric extraction results

Bunch investigation itself isn't one explicit calculation, yet the general errand to be illuminated. It tends to be accomplished by different calculations that contrast essentially in their comprehension of what compromise a group and how to effectively discover them.

Mainstream thoughts of bunches incorporate gatherings with little separations between group individuals, thick zones of the information space, interims or specific factual circulations. Grouping can along these lines be defined as a multi-target improvement issue.

In the next section of the work, the achieved results from the proposed algorithm is elaborated.

\section{RESULTS \& DISCUSSIONS}

The proposed algorithm are evaluated on standard and benchmark datasets[12] and the results are highly satisfactory. In this section of the work, the obtained results

Firstly, the results from the parametric extraction part of the algorithm is elaborated here[Table-1]

\section{TABLE -I}

\& Sciences Publication Exploring Innovation


As a speculation of this down to earth depiction, the arithmetic of differential math characterizes the slope of a bend at a point as the slope of the digression line by then. At the point when the bend is given by a progression of focuses in a graph or in a graph or in a rundown of the directions of focuses, the slope might be determined not at a point however between any two given focuses. At the point when the bend is given as a nonstop capacity, maybe as an arithmetical equation, at that point the differential analytics gives rules giving a recipe to the slope of the bend anytime in the bend.A measurement space is a set for which separates between all individuals from the set are characterized. Along these lines, a wide range of sort of separations can be determined, for example, for traversal of diagrams, examination of conveyances and bends, and utilizing bizarre meaning of space.

Two unmistakable planes can either meet in atypical line or are parallel. Three planes, no pair of which are parallel, can either meet in a typical line, meet in a remarkable normal point or share no point for all intents and purpose. In the last case, the three lines of convergence of each pair of planes are commonly parallel.

Territory is the amount that communicates the degree of a two-dimensional figure or shape lamina, in the plane. Surface region is its simple on the two-dimensional surface of a three- dimensional item. Zone can be comprehended as the measure of material with a given of the shape, or the measure of paint important to cover the surface with a solitary coat.

The real time extraction results are visualized graphically here[fig-3 to Fig-6]
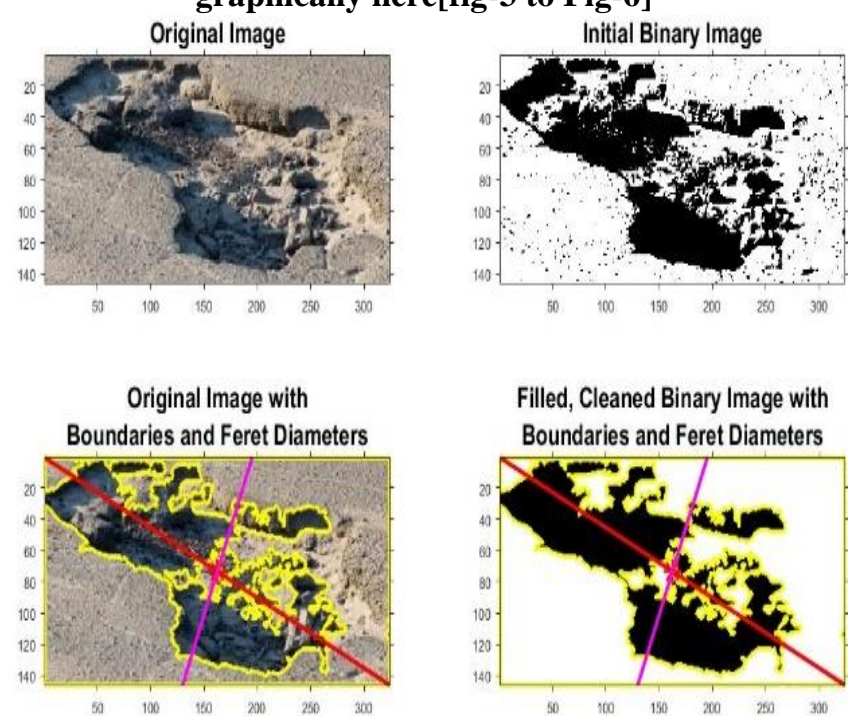

Fig. 2 Parametric Extraction of the Sample Set - 1
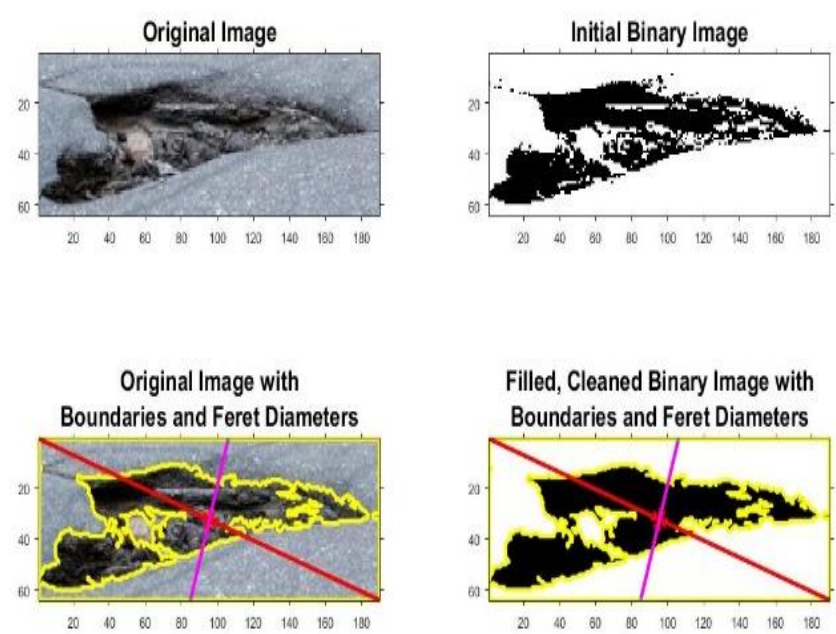

Filled, Cleaned Binary Image with Boundaries and Feret Diameters

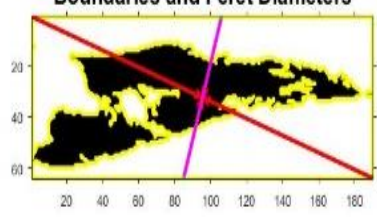

Fig. 3 Parametric Extraction of the Sample Set - 2
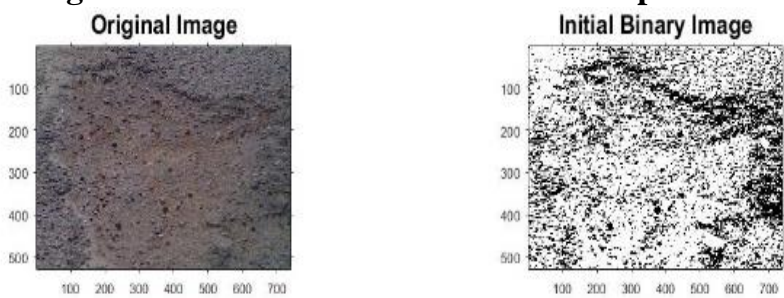

Original Image with Boundaries and Feret Diameters

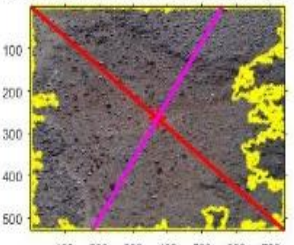

Filled, Cleaned Binary Image with Boundaries and Feret Diameters

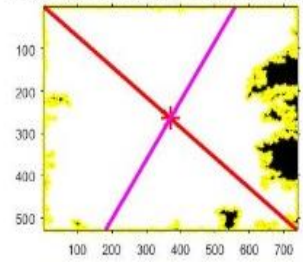

Fig. 4 Parametric Extraction of the Sample Set -3
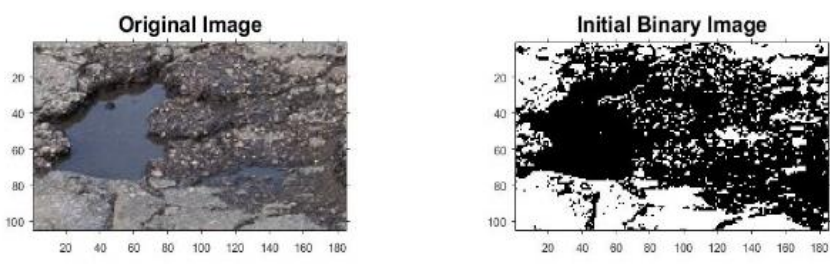

Original Image with Boundaries and Feret Diameters

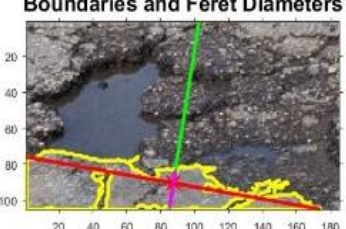

Filled, Cleaned Binary Image with Boundaries and Feret Diameters

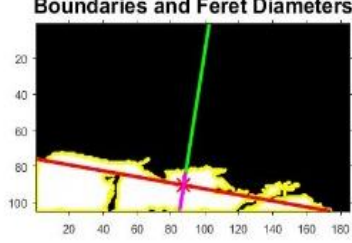

Fig. 5 Parametric Extraction of the Sample Set -4

Secondly, the cluster analysis results are also furnished here [Table - 2]. 
TABLE II

Cluster ANAlysis Results

\begin{tabular}{|l|l|l|l|l|}
\hline Elements & $\begin{array}{l}\text { Complete } \\
\text { Data }\end{array}$ & $\begin{array}{l}\text { Cluster - 1 } \\
\text { (Centroids) }\end{array}$ & $\begin{array}{l}\text { Cluster - 2 } \\
\text { (Centroids) }\end{array}$ & $\begin{array}{l}\text { Cluster - 3 } \\
\text { (Centroids) }\end{array}$ \\
\hline Long Slope & 0.2931 & 0.366 & -0.2175 & 0.7125 \\
\hline $\begin{array}{l}\text { Perpendicular } \\
\text { Slope }\end{array}$ & -0.2492 & -3.408 & 4.87 & -1.42 \\
\hline $\begin{array}{l}\text { Defect } \\
\text { Number }\end{array}$ & 1.3077 & 1.4 & 1.25 & 1.25 \\
\hline $\begin{array}{l}\text { Max Distance } \\
\text { of the Defect }\end{array}$ & 496.9362 & 234.778 & 468.9575 & 852.6125 \\
\hline $\begin{array}{l}\text { Depth of the } \\
\text { defect }\end{array}$ & 320.0131 & 105.952 & 190 & 717.6025 \\
\hline $\begin{array}{l}\text { Average } \\
\text { Width }\end{array}$ & 145.8323 & 46.884 & 104.18 & 311.17 \\
\hline Area & 112269.9231 & 12063.2 & 71715.25 & 278083 \\
\hline Class & Defects & Cracks & Patch & Pothole \\
\hline
\end{tabular}

The results are visualized graphically here [Fig - 7].

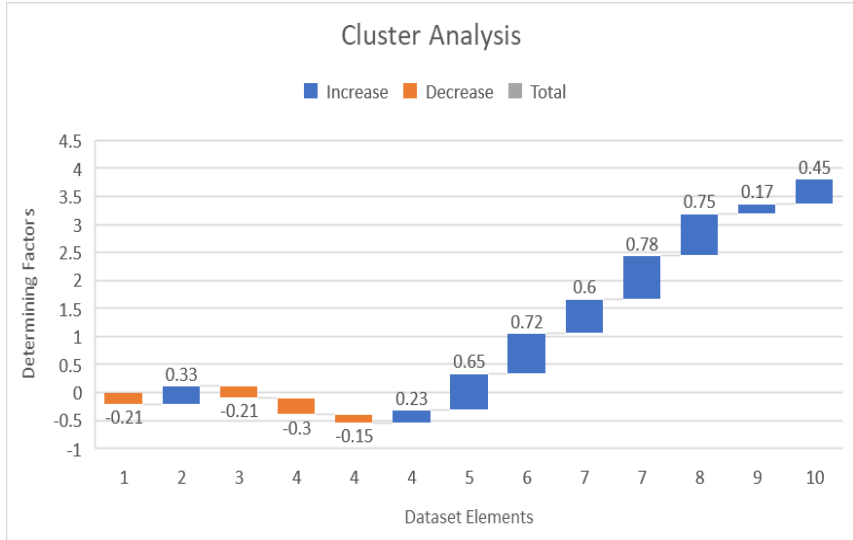

Fig. 6 Cluster Analysis

Finally, the accuracy analysis is carried out with the benchmark dataset[Table-3].

TABLE III

ACCURACy ANALysis

\begin{tabular}{|c|c|c|c|}
\hline $\begin{array}{c}\text { Dataset Element } \\
\text { ID }\end{array}$ & $\begin{array}{c}\text { Defect } \\
\text { Number }\end{array}$ & $\begin{array}{c}\text { Actual Defect } \\
\text { Type }\end{array}$ & $\begin{array}{c}\text { Identified } \\
\text { Defect Type }\end{array}$ \\
\hline 1 & 1 & Patchwork & Patchwork \\
\hline 2 & 1 & Pothole & Pothole \\
\hline 3 & 1 & Crack & Pothole \\
\hline 4 & 1 & Crack & Crack \\
\hline 4 & 2 & Pothole & Pothole \\
\hline 4 & 3 & Pothole & Crack \\
\hline 5 & 1 & Crack & Crack \\
\hline 6 & 1 & Patchwork & Patchwork \\
\hline 7 & 1 & Patchwork & Patchwork \\
\hline 7 & 2 & Patchwork & Patchwork \\
\hline 8 & 1 & Pothole & Pothole \\
\hline 9 & 1 & Patchwork & Patchwork \\
\hline 10 & 1 & Crack & Crack \\
\hline
\end{tabular}

For the sample size of 10 data items, the proposed algorithm demonstrates nearly $85 \%$ accuracy and for the complete dataset of 100 data items, the obtained accuracy is nearly $96 \%$.Henceforth, after the detailed analysis of the results, in the next section of the work, the comparative analysis is presented.

\section{COMPARATIVE ANALYSIS}

After the detailed discussion on the algorithm and results obtained from the proposed algorithm, in this section of the work, the proposed methods are compared with the parallel research outcomes[Table-4]

TABLE -IV

COMPARATIVE ANALYSIS

\begin{tabular}{|c|c|c|}
\hline Research Outcome & Methods Applied & Accuracy (\%) \\
\hline S. Kanarachos et al. [5] & $\begin{array}{l}\text { - Deep Learning } \\
\text { - Parameter Extraction } \\
\text { - Expert Systems }\end{array}$ & $89 \%$ \\
\hline H. Bello-Salau et al. [6] & $\begin{array}{l}\text { - Parameter Extraction } \\
\text { - Sensor network } \\
\text { - Data accumulation }\end{array}$ & $92 \%$ \\
\hline F. M. Bayer et al. [7] & $\begin{array}{l}\text { - Signal Processing } \\
\text { - } \text { Data Normalization } \\
\end{array}$ & $90 \%$ \\
\hline K. Azhar et al. [8] & $\begin{array}{l}\text { - Location aggregation } \\
\text { - Image Segmentation }\end{array}$ & $88 \%$ \\
\hline PE-PC-DD & $\begin{array}{ll}\text { - } & \text { Parameter Extraction } \\
\text { - } & \text { Pragmatic Clustering } \\
\text { - } & \text { DefectCategory } \\
& \text { Separation } \\
\end{array}$ & $96 \%$ \\
\hline
\end{tabular}

For some random division of a picture, this plan yields the quantity of bits required to encode that picture dependent on the given division. Accordingly, among every single imaginable division of a picture, the objective is to discover the division which delivers the briefest coding length.

This can be accomplished by a basic agglomerative grouping strategy. The mutilation in the lossy pressure decides the coarseness of the division and its ideal worth may contrast for each picture.This parameter can be assessed heuristically from the complexity of surfaces in a picture are comparative, for example in cover picture, more grounded affectability and along these lines lower quantization is required.

\section{CONCLUSION}

The growth in the road network in across all the places in India have motivated multiple researcher to automate the road maintenance activities. The road surface defects can be distinguished in few categories as crakes, potholes and patchwork. During the study this work, identifies that the extraction of the study this work, identifies that the extraction of the relevant parameters for the severity detection in the parallel research outcomes are missing and also the unsupervised learning methods are highly expected in case of the defect detection. Henceforth, this work deploys a set of models for the automatic identification of the road surface defects. Firstly, extraction of the coordinate values from the pixel information is used to determine the maximal and minimal distance between the objects in the road condition information. This helps in finding the surrounding information of the objects. Secondly, extraction of the intensity variation is used to produce the depth of the defects using the centroid estimation in the road condition information. 
Thirdly, the width of the defect can be calculated using maxima and minima points in the road condition information, this intern helps to develop the surface area of the defects. Fourthly, the area of the defect is used to be calculated using the coordinate vector from the road condition information. Finally, in the absence of the class determination factors, the pragmatic calculation of the ratio-based coefficients can form the determining factors, which intern generate the categorization of the defect and finally makes the repair process automate for making the road transport much safer and fast and better.

\section{REFERENCES}

1. European Commission, Mobility and transport road safety in the EuropeanUnion: trends statistics and main challenges, Luxembourg: Publications Office of the European Union, 2018.

2. C. N. do Transports, Pesquisa ConfederaÁ,,

ONacional do Transports (CNT) de Rodovias 2018: relatÛriogerencial, 2018.

3. A. Fox, B. V. K. Vijaya-Kumar, J. Chen, F. Bai, "Multi-lane potholedetection from crowdsourced undersampled vehicle sensor data", IEEE Transactions on Mobile Computing, vol. 16, no. 12, pp. 3417-3430, 2017.

4. T. Kim, S. Ryu, "Review and analysis of pothole detection methods",Journal of Emerging Trends in Computing and Information Sciences, vol. 5, no. 8, 2014.

5. S. Kanarachos, S.-R. G. Christopoulos, A. Chroneos, M. E. Fitzpatrick, "Detecting anomalies in time series data via a deep learning algorithm combining wavelets neural networks and hilbert transform", Expert Systems with Applications, vol. 85, pp. 292-304, 2017.

6. H. Bello-Salau, A. M. Aibinu, A. J. Onumanyi, E. N. Onwuka, J. J. Dukiya, H. Ohize, "New road anomaly detection and characterization algorithm for autonomous vehicles", Applied Computing and Informatics, 2018, [online] Available: https://doi.org/10.1016/j.aci.2018.05.002.

7. Wavelets: Principles Analysis and Applications, Joseph Burgess, 2018.

8. F. M. Bayer, A. J. Kozakevicius, R. J. Cintra, "An iterative waveletthreshold for signal denoising", Signal Processing, vol. 162, pp. 10-20, September 2019.

9. K. Azhar, F. Murtaza, M. H. Yousaf, H. A. Habib, "Computer visionbased detection and localization of potholes in asphalt pavement images", 2016 IEEE Canadian Conference on Electrical and Computer Engineering (CCECE), pp. 1-5, May 2016.

10. P. Wang, Y. Hu, Y. Dai, M. Tian, "Asphalt pavement pothole detection and segmentation based on wavelet energy field", Mathematical Problems in Engineering, vol. 2017, 2017.

11. H. Maeda, Y. Sekimoto, T. Seto, H. Omata, "Road damage detectionand classification using deep neural networks with smartphone images: Road damage detection and classification", Computer-Aided Civil and Infrastructure Engineering, vol. 33, no. 2, July 2018.

12. Dataset: Indian Surface Maintenance

13. Authority Image Samples.

\section{AUTHORS PROFILE}

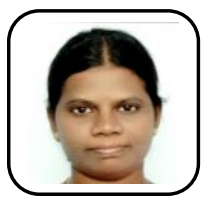

Anitha Kumar Dara Research scholor, in Department of Computer Science and Engineering ,JNTUH CEH, Hyderabad, Telangana, India . Interested areas in Data Mining, Machine Learning and Image processing.anithakumaridara@gmail.com

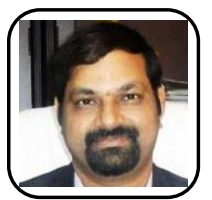

Dr. A. Govardhan B.E.(CSE), MTech (CS), Ph.D.(CSE)

Professor, Registrar\& Rector, JNTUH Hyderabad, Telangana, India, FIE, SMCSI, MACM, MIEEE, MIAENG, MWASET, LMISTE, MFSF, MTDWI, SMIACSIT Computer Science \& Engineering,Dr.A.Govardhan is presently a Professor of Computer Science \& Engineering, Registrar and Executive Council Member,
Jawaharlal Nehru Technological University Hyderabad (JNTUH). He served and held several Academic and Administrative positions including Principal (JNTUH CEH), Director (School of Information Technology, JNTUH), Director of Evaluation (JNTUH), Principal(JNTUH CEJ), Head of the Department, Chairman and Member of Boards of Studies and Students' Advisor. He did his 10th standard from Z.P.H.S. Choutuppal in 1986, Intermediate from Andhra Pradesh Residential Junior College (APRJC), Nagarjuna Sagar in 1988, B.E.(CSE) from Osmania University College of Engineering, Hyderabad in 1992, M.Tech from Jawaharlal Nehru University(JNU), New Delhi in 1994 and Ph.D from Jawaharlal Nehru Technological University, Hyderabad in 2003. He is the recipient of 33 International and National Awards including Best Senior Scientist Award (2018), Dewang Mehta Academic Leadership Award (2017), Dr.Sarvepalli Radhakrishnan National Award, A.P. State Government Best Teacher Award (2012), Pride of Asia International Award, Best Principal, Bharat Seva Ratna Puraskar, CSI Chapter Patron Award, Bharat Jyoti Award, International Intellectual Development Award and Mother Teresa Award for Outstanding Services, Achievements, Contributions, Meritorious Services, Outstanding Performance and Remarkable Role in the field of Education and Service to the Nation. He is a Chairman and Member on several Boards of Studies of various Universities. He is the Past Chairman and Vice-Chairman of CSI Hyderabad Chapter. He is a Member on the Editorial Boards for Twelve International Journals.

He is a Member on Advisory Boards \& Academic Boards and Technical Program Committee Member for more than 85 International and National Conferences. He is Member on Boards of Governors and Academic Councils for number of colleges. He is an Editor for 4 Springer Proceedings.He has 3 Monographs, and 10 Book Chapters in Springer, Germany. He has guided 85 Ph.D theses, 1 M.Phil and 135 M.Tech projects He has published 555 research papers at International/National Journals/Conferences including IEEE, ACM, Springer, Elsevier and InderScience. He is an Editor for 4 Springer Proceedings. He has organized 4 International Conferences, 20 Workshops and 1 Refresher Course. He has delivered more than 100 Keynote speeches and invited lectures. He has Chaired 22 Sessions at the International/ National Conferences in India and Abroad. He has the Research Projects (Completed/ Ongoing) worth of Rs. 1.159 Crores. He has 25 years of Teaching and Research experience. He served as a Convener for TS ECET-2019,TS ECET-2018, TS ECET-2017, Co-Convener for TS EAMCET-III-2016, EAMCET-2009 and Chief Regional Coordinator for TS EAMCET-2016, EAMCET-2010 and EAMCET-2011. He has also served as Chairman \& Vice-Chairman, CSI Hyderabad Chapter and IT professional Forum, A.P. He is a member on several Professional and Service Oriented Bodies. His areas of research include Databases, Data Science and Information Retrieval Systems. 